\title{
Pseudo-3D Subsurface Imaging of Pharmaceutical Solid Dosage Forms Using Micro-spatially Offset Low-Frequency Raman Spectroscopy
}

\author{
Kārlis Bērziņšs ${ }^{\mathrm{a}}$, Sara J. Fraser-Miller ${ }^{\mathrm{a}}$, Keith C. Gordon ${ }^{\mathrm{a}^{*}}$ \\ aThe Dodd-Walls Centre for Photonic and Quantum Technologies, Department of Chemistry, \\ University of Otago, Dunedin 9016, New Zealand \\ *Corresponding author (keith.gordon@otago.ac.nz)
}

\section{Supporting information}

\section{Table of contents}

Table S1. Information on the characteristics of the model pharmaceutical tablets.

Figure S1. Digital images of the cross-sections of $200 \mathrm{mg}$ bilayer tablets with varied celecoxib form III and PVP content $(\mathrm{w} / \mathrm{w})$.

Figure S2. Digital images of the cross-sections of bilayer tablets with constant celecoxib form III, but varied PVP content $(\mathrm{w} / \mathrm{w})$. S-3

Figure S3. Digital images of the cross-sections of $200 \mathrm{mg}$ trilayer tablets with varied celecoxib form III, $\alpha$-LM and PVP content (w/w/w).....

Figure S4. Resolved MCR loadings spectra of the two components using (a) LFR and (b) MFR microspatially offset data that were recorded for celecoxib form III and PVP bilayer tablets. .................. S-4 Figure S5. Signal to noise ratio (SNR) analysis of celecoxib form III and PVP bilayer tablets of different composition (w/w) using (a) LFR or (b) MFR data recorded at different sample displacements from

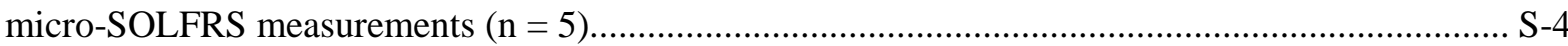
Figure S6. A comparison between the PVP layer thickness and layer weight for different bilayer tablets

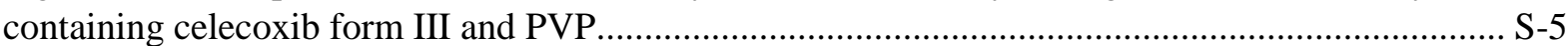
Figure S7. Representative loadings plots of factors 2 and 3 of PLS regression model for PVP layer thickness determination within celecoxib and PVP $200 \mathrm{mg}$ bilayer tablets using MFR micro-spatially offset spectroscopic data collected at $4.0 \mathrm{~mm}$ defocussing distance.

Table S2. Summary of the performance for the PLS regression models created using either LFR or MFR micro-spatially offset spectroscopic data for $200 \mathrm{mg}$ bilayer tablets containing celecoxib form III and

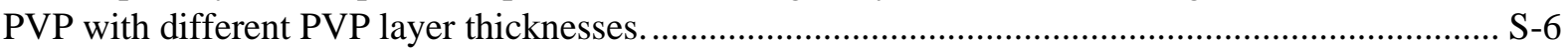
Table S3. Summary of the performance for the PLS regression models created using either LFR or MFR micro-spatially offset spectroscopic data for bilayer tablets with constant celecoxib form III, but varied PVP content.

Table S4. Summary of the performance for the global PLS regression models created using either LFR or MFR micro-spatially offset spectroscopic data for bilayer tablets with both constant and varied celecoxib form III content.

Figure S9. Examples of (a) low and (b) mid-frequency micro-spatially offset Raman spectra for celecoxib form III, $\alpha$-LM and PVP trilayer tablet (1:2:1 composition; 50/100/50 mg)................. S-10 Table S5. Summary of the performance for the PLS regression models created using either LFR or MFR micro-spatially offset spectroscopic data for trilayer tablets with varied celecoxib form III, $\alpha$-LM and PVP content.

Figure S11. Examples of raw spectra collected at different sample displacements for the same areas of a multi-component homogenous tablet containing celecoxib, $\alpha$-LM and PVP dehydrated ( $\alpha$-LM to $\alpha$ $\mathrm{Ls})$ at $140{ }^{\circ} \mathrm{C}$ for 20 minutes. S-13

Figure S12. PC1 loadings plot for PCA of micro-SOLFRS mapping data collected from multicomponent tablet containing celecoxib, $\alpha$-LM and PVP dehydrated ( $\alpha$-LM to $\left.\alpha-L_{S}\right)$ at $140{ }^{\circ} \mathrm{C}$ for 20 minutes. The red labels denote unique peaks attributed to celecoxib form III. 
Table S1. Information on the characteristics of the model pharmaceutical tablets.

\begin{tabular}{|c|c|c|c|c|c|}
\hline Components & $\begin{array}{c}\text { Tablet } \\
\text { weight, mg }\end{array}$ & $\begin{array}{l}\text { Component } \\
\text { ratio }\end{array}$ & $\begin{array}{c}\text { Tablet } \\
\text { thickness, } \\
\mathrm{mm}\end{array}$ & $\begin{array}{l}\text { PVP layer } \\
\text { thickness, } \mu \mathrm{m}\end{array}$ & $\begin{array}{c}\alpha \text {-LM layer } \\
\text { thickness, } \mu \mathrm{m}\end{array}$ \\
\hline \multirow{14}{*}{ Celecoxib : PVP } & \multirow{9}{*}{200} & $1: 9$ & 3.35 & 3110 & N/A \\
\hline & & $1: 4$ & 3.25 & 2740 & N/A \\
\hline & & $3: 7$ & 3.25 & 2508 & N/A \\
\hline & & $2: 3$ & 3.20 & 2175 & N/A \\
\hline & & $1: 1$ & 2.95 & 1785 & N/A \\
\hline & & $3: 2$ & 3.05 & 1597 & N/A \\
\hline & & $7: 3$ & 2.90 & $-\mathrm{-a}^{\mathrm{a}}$ & N/A \\
\hline & & $4: 1$ & 2.90 & - & N/A \\
\hline & & $9: 1$ & 2.85 & - & N/A \\
\hline & 280 & $5: 9$ & 4.20 & 3301 & N/A \\
\hline & 260 & $5: 8$ & 4.10 & 3002 & N/A \\
\hline & 240 & $5: 7$ & 3.75 & 2553 & N/A \\
\hline & 220 & $5: 6$ & 3.45 & 2283 & N/A \\
\hline & 180 & $5: 4$ & 2.75 & 1437 & N/A \\
\hline \multirow{6}{*}{$\begin{array}{l}\text { Celecoxib : } \alpha- \\
\text { LM : PVP }\end{array}$} & \multirow{6}{*}{200} & $1: 1: 2$ & 3.20 & 1902 & 528 \\
\hline & & $2: 3: 3$ & 3.00 & 1447 & 894 \\
\hline & & $1: 2: 1$ & 2.95 & 1301 & 899 \\
\hline & & $3: 3: 2$ & 2.95 & 857 & 947 \\
\hline & & $2: 1: 1$ & 2.90 & 941 & 720 \\
\hline & & $1: 6: 1^{b}$ & 2.95 & N/A & N/A \\
\hline
\end{tabular}

${ }^{\mathrm{a}}$ The brittle nature of the thin upper layer disallowed (accurate) layer thickness measurements.

${ }^{\mathrm{b}}$ Homogenous mixture. 


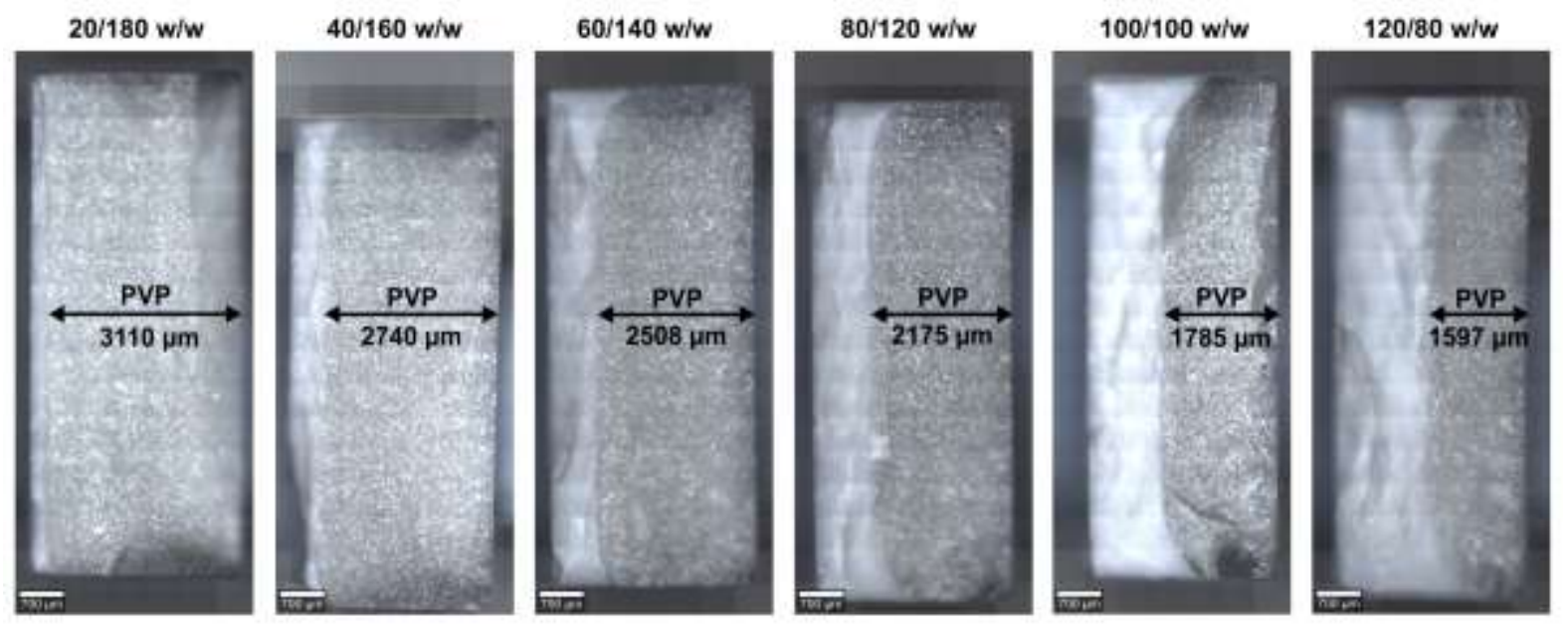

Figure S1. Digital images of the cross-sections of $200 \mathrm{mg}$ bilayer tablets with varied celecoxib form III and polyvinylpyrrolidone (PVP) content (w/w).

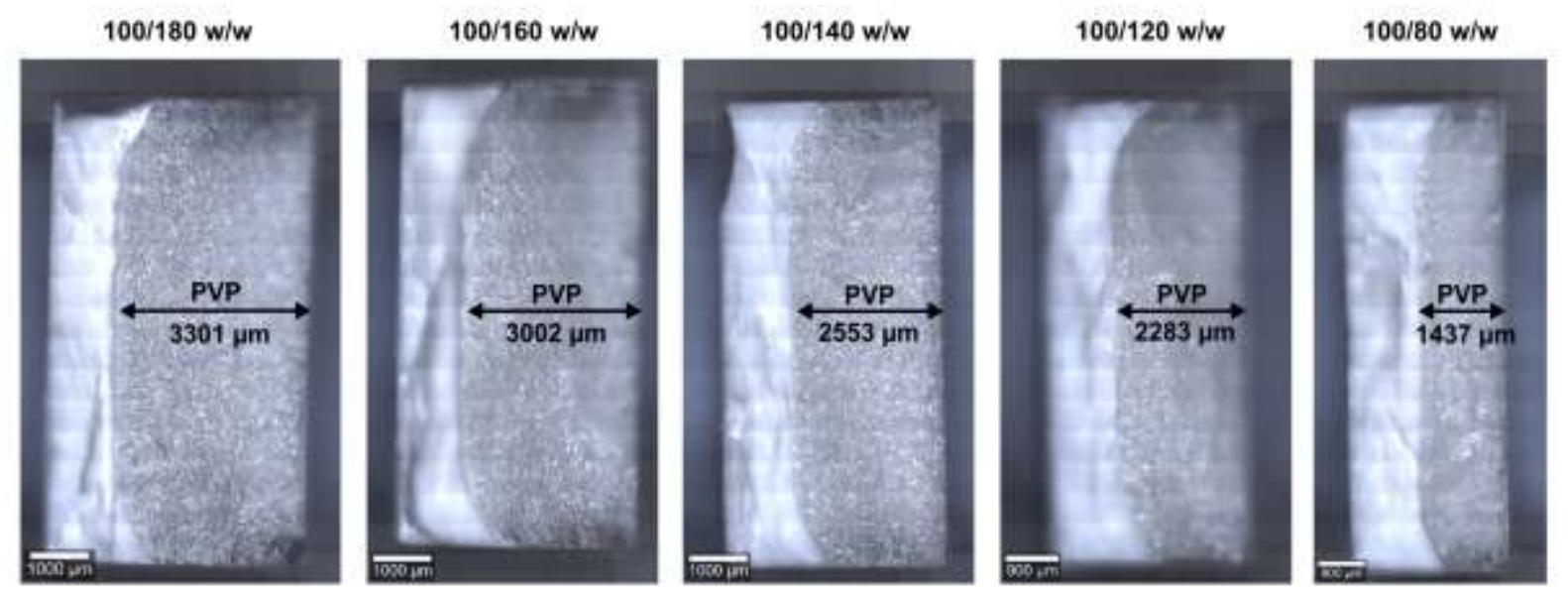

Figure S2. Digital images of the cross-sections of bilayer tablets with constant celecoxib form III, but varied polyvinylpyrrolidone (PVP) content (w/w).
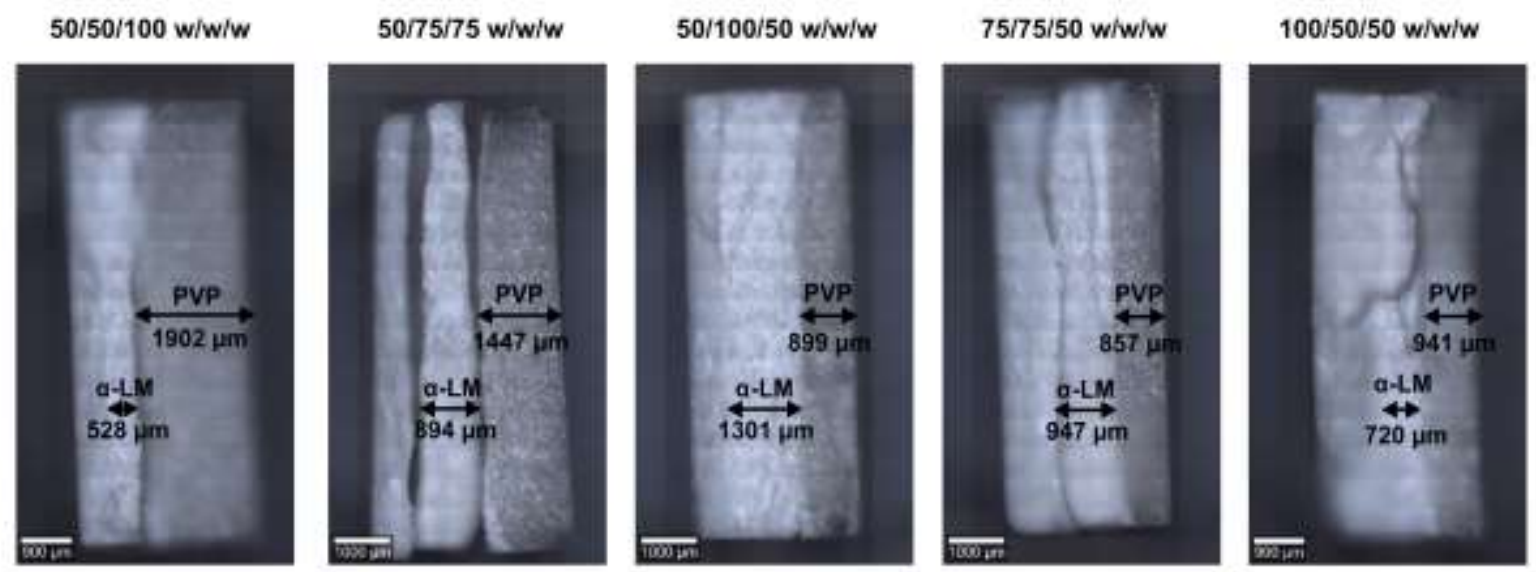

Figure S3. Digital images of the cross-sections of $200 \mathrm{mg}$ trilayer tablets with varied celecoxib form III, $\alpha$-lactose monohydrate ( $\alpha$-LM) and polyvinylpyrrolidone (PVP) content (w/w/w). 

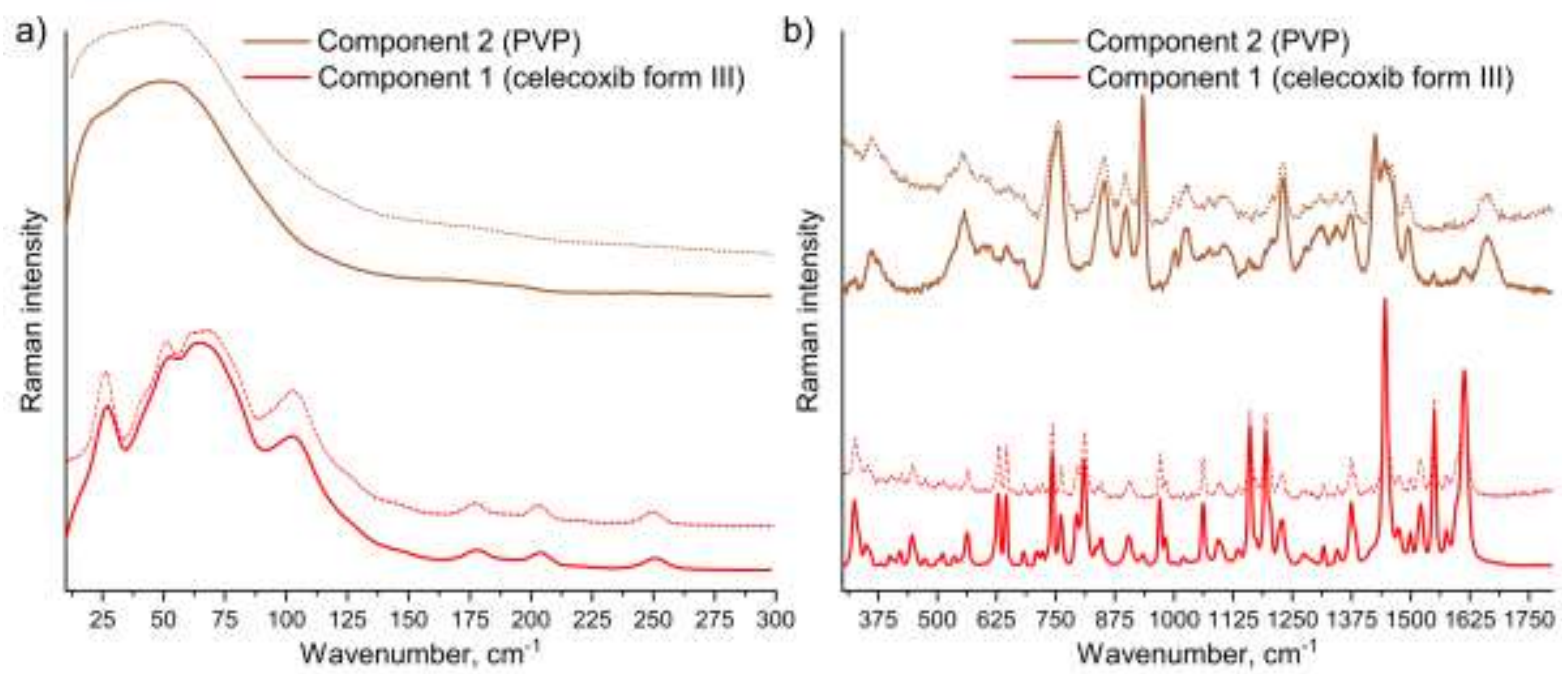

Figure S4. Resolved MCR loadings spectra of the two components using (a) LFR and (b) MFR microspatially offset data that were recorded for celecoxib form III and polyvinylpyrrolidone (PVP) bilayer tablets. Dashed line spectra denote the Raman signature of the corresponding chemical entity to the MCR component for a visual reference.
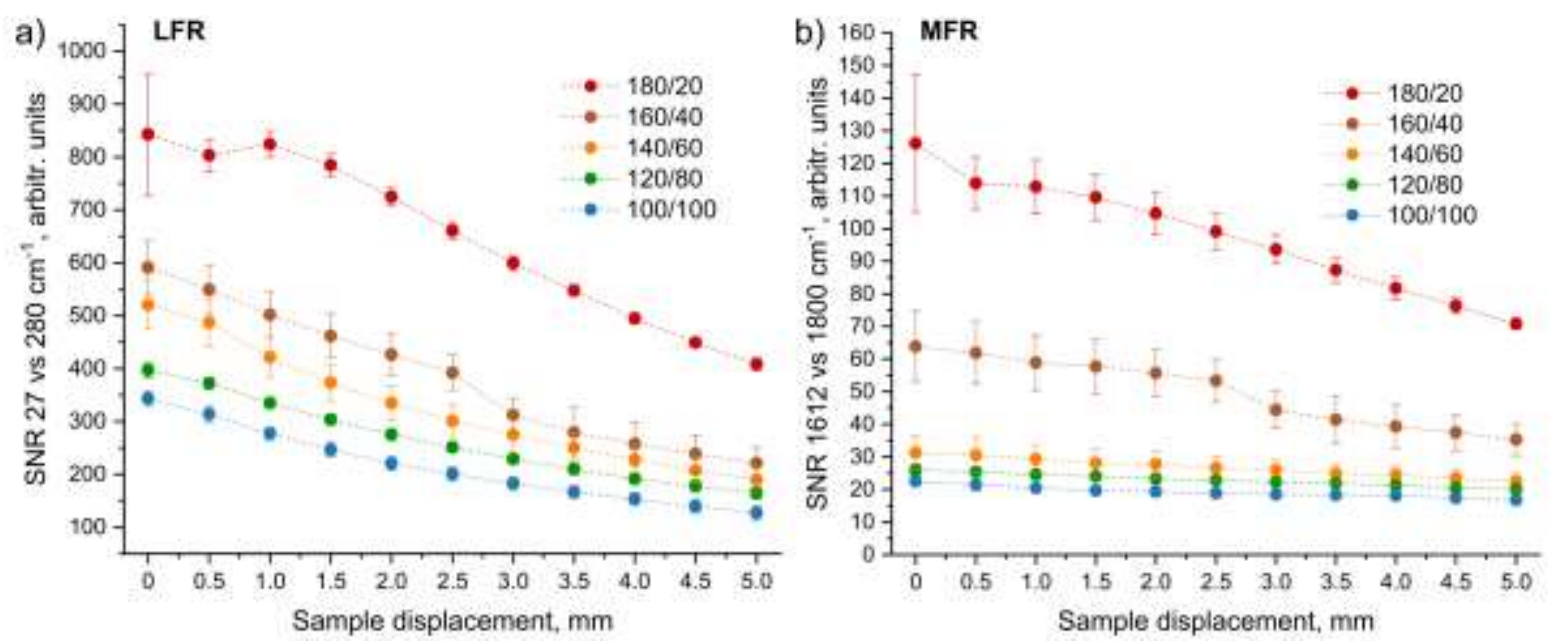

Figure S5. Signal to noise ratio (SNR) analysis of celecoxib form III and PVP bilayer tablets of different composition (w/w) using (a) LFR or (b) MFR data recorded at different sample displacements from micro-SOLFRS measurements $(\mathrm{n}=5)$. SNR (where applicable) was calculated using the first standard deviation (FSD) approach by dividing the intensity difference between the peak of interest (27 or 1612 $\mathrm{cm}^{-1}$ ) and selected background (280 or $1800 \mathrm{~cm}^{-1}$ ) with the square root of the respective background signal intensity. The lines are drawn to assist in visualizing the trends. 


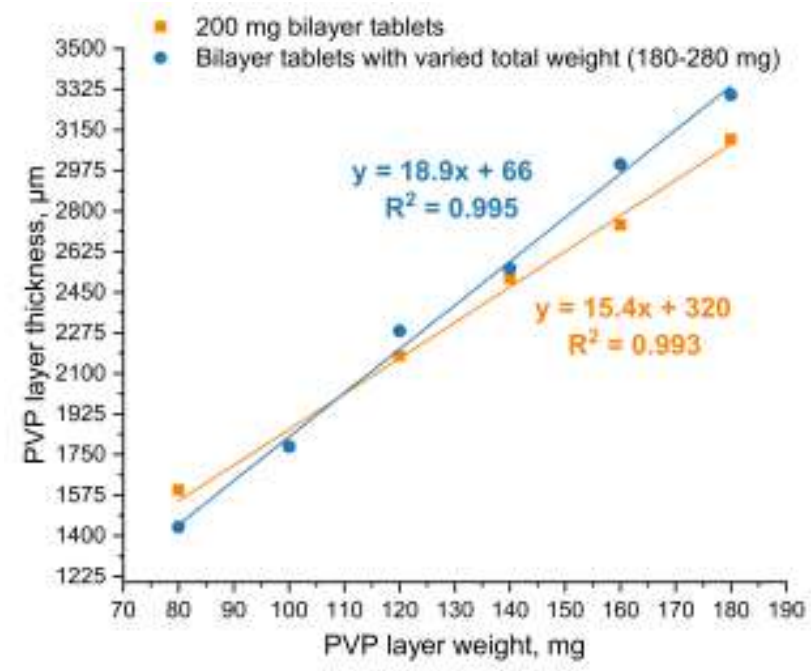

Figure S6. A comparison between the PVP layer thickness and layer weight for different bilayer tablets containing celecoxib form III and PVP.

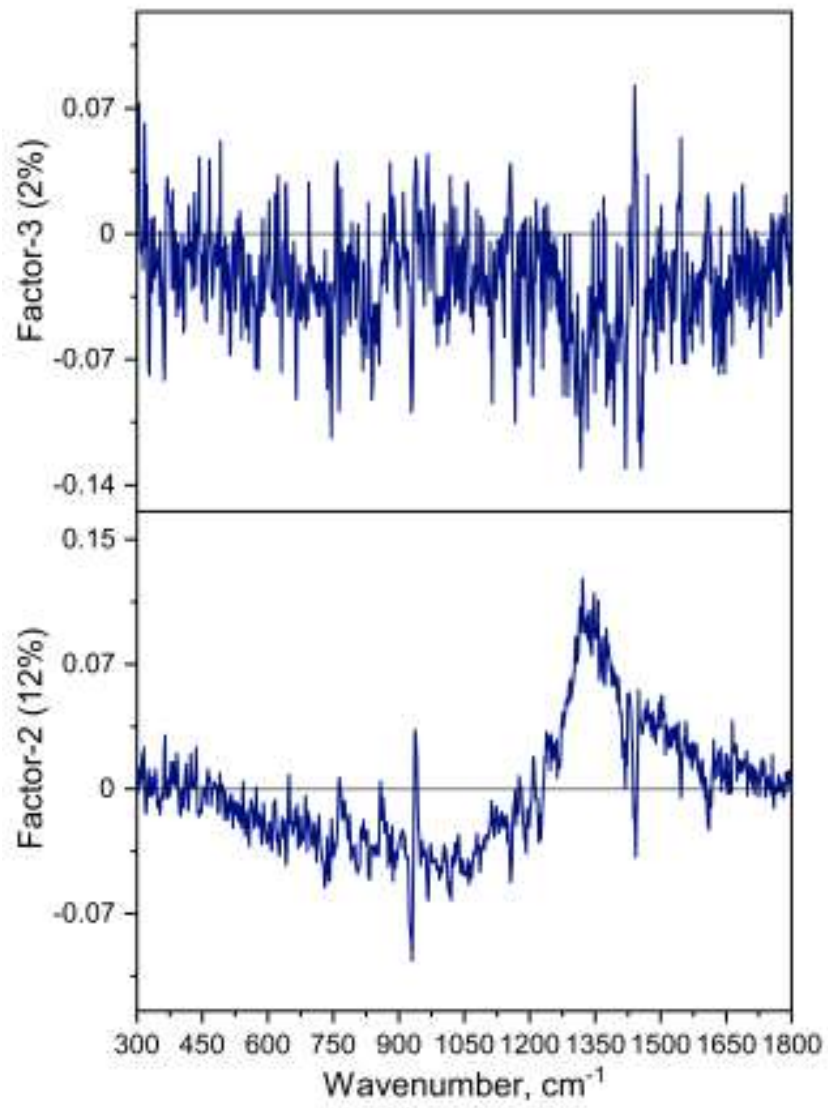

Figure S7. Representative loadings plots of factors 2 and 3 of PLS regression model for PVP layer thickness determination within celecoxib and PVP $200 \mathrm{mg}$ bilayer tablets using MFR micro-spatially offset spectroscopic data collected at $4.0 \mathrm{~mm}$ defocusing distance. 
Table S2. Summary of the performance for the PLS regression models created using either LFR or MFR micro-spatially offset spectroscopic data for 200 mg bilayer tablets containing celecoxib form III and PVP with different PVP layer thicknesses.

\begin{tabular}{|c|c|c|c|c|c|c|c|c|c|c|c|c|c|c|}
\hline & & \multicolumn{5}{|c|}{ Calibration } & \multicolumn{4}{|c|}{ Cross validation } & \multicolumn{4}{|c|}{ Test set } \\
\hline & Sample displacement, $\mathrm{mm}$ & Factors & Slope & Offset & RMSEC & $\mathrm{R}^{2}$ & Slope & Offset & RMSECV & $\mathrm{R}^{2}$ & Slope & Offset & RMSEP & $\mathrm{R}^{2}$ \\
\hline \multirow{11}{*}{ LFR } & 0 & \multirow{11}{*}{3} & 0.98 & 33 & 63 & 0.98 & 0.97 & 67 & 87 & 0.97 & 0.91 & 188 & 96 & 0.97 \\
\hline & 0.5 & & 0.99 & 24 & 54 & 0.99 & 0.98 & 60 & 79 & 0.98 & 0.96 & 111 & 95 & 0.97 \\
\hline & 1.0 & & 0.97 & 59 & 84 & 0.97 & 0.95 & 112 & 119 & 0.95 & 0.99 & 38 & 114 & 0.95 \\
\hline & 1.5 & & 0.98 & 47 & 75 & 0.98 & 0.96 & 103 & 105 & 0.96 & 0.89 & 241 & 112 & 0.96 \\
\hline & 2.0 & & 0.98 & 50 & 77 & 0.98 & 0.95 & 122 & 111 & 0.96 & 0.99 & -4.5 & 76 & 0.98 \\
\hline & 2.5 & & 0.99 & 29 & 59 & 0.99 & 0.96 & 96 & 96 & 0.97 & 0.95 & 92 & 71 & 0.98 \\
\hline & 3.0 & & 0.98 & 43 & 72 & 0.98 & 0.95 & 126 & 109 & 0.96 & 0.96 & 66 & 74 & 0.98 \\
\hline & 3.5 & & 0.99 & 28 & 58 & 0.99 & 0.95 & 111 & 95 & 0.97 & 0.97 & 71 & 83 & 0.98 \\
\hline & 4.0 & & 0.99 & 21 & 50 & 0.99 & 0.96 & 105 & 86 & 0.98 & 0.93 & 136 & 90 & 0.97 \\
\hline & 4.5 & & 0.99 & 21 & 50 & 0.99 & 0.96 & 90 & 79 & 0.98 & 0.90 & 232 & 113 & 0.96 \\
\hline & 5.0 & & 0.99 & 24 & 54 & 0.99 & 0.94 & 133 & 105 & 0.96 & 0.93 & 153 & 99 & 0.97 \\
\hline \multirow{11}{*}{ MFR } & 0 & \multirow{11}{*}{1} & 0.83 & 396 & 218 & 0.83 & 0.80 & 454 & 247 & 0.80 & 0.83 & 398 & 218 & 0.83 \\
\hline & 0.5 & & 0.82 & 409 & 221 & 0.82 & 0.80 & 469 & 249 & 0.80 & 0.79 & 486 & 231 & 0.81 \\
\hline & 1.0 & & 0.83 & 402 & 219 & 0.83 & 0.80 & 463 & 245 & 0.81 & 0.82 & 416 & 217 & 0.83 \\
\hline & 1.5 & & 0.84 & 371 & 211 & 0.84 & 0.81 & 426 & 236 & 0.82 & 0.82 & 414 & 219 & 0.83 \\
\hline & 2.0 & & 0.84 & 364 & 209 & 0.84 & 0.82 & 424 & 233 & 0.83 & 0.83 & 397 & 216 & 0.83 \\
\hline & 2.5 & & 0.84 & 370 & 211 & 0.84 & 0.81 & 429 & 235 & 0.82 & 0.83 & 401 & 209 & 0.84 \\
\hline & 3.0 & & 0.83 & 400 & 219 & 0.83 & 0.80 & 468 & 244 & 0.81 & 0.81 & 431 & 220 & 0.82 \\
\hline & 3.5 & & 0.85 & 355 & 206 & 0.85 & 0.82 & 415 & 230 & 0.83 & 0.84 & 361 & 211 & 0.84 \\
\hline & 4.0 & & 0.85 & 357 & 207 & 0.85 & 0.82 & 421 & 232 & $\mathbf{0 . 8 3}$ & 0.85 & 351 & 205 & 0.85 \\
\hline & 4.5 & & 0.85 & 346 & 204 & 0.85 & 0.82 & 412 & 228 & 0.83 & 0.85 & 341 & 209 & 0.84 \\
\hline & 5.0 & & 0.85 & 357 & 207 & 0.85 & 0.82 & 424 & 232 & 0.83 & 0.85 & 351 & 212 & 0.84 \\
\hline
\end{tabular}



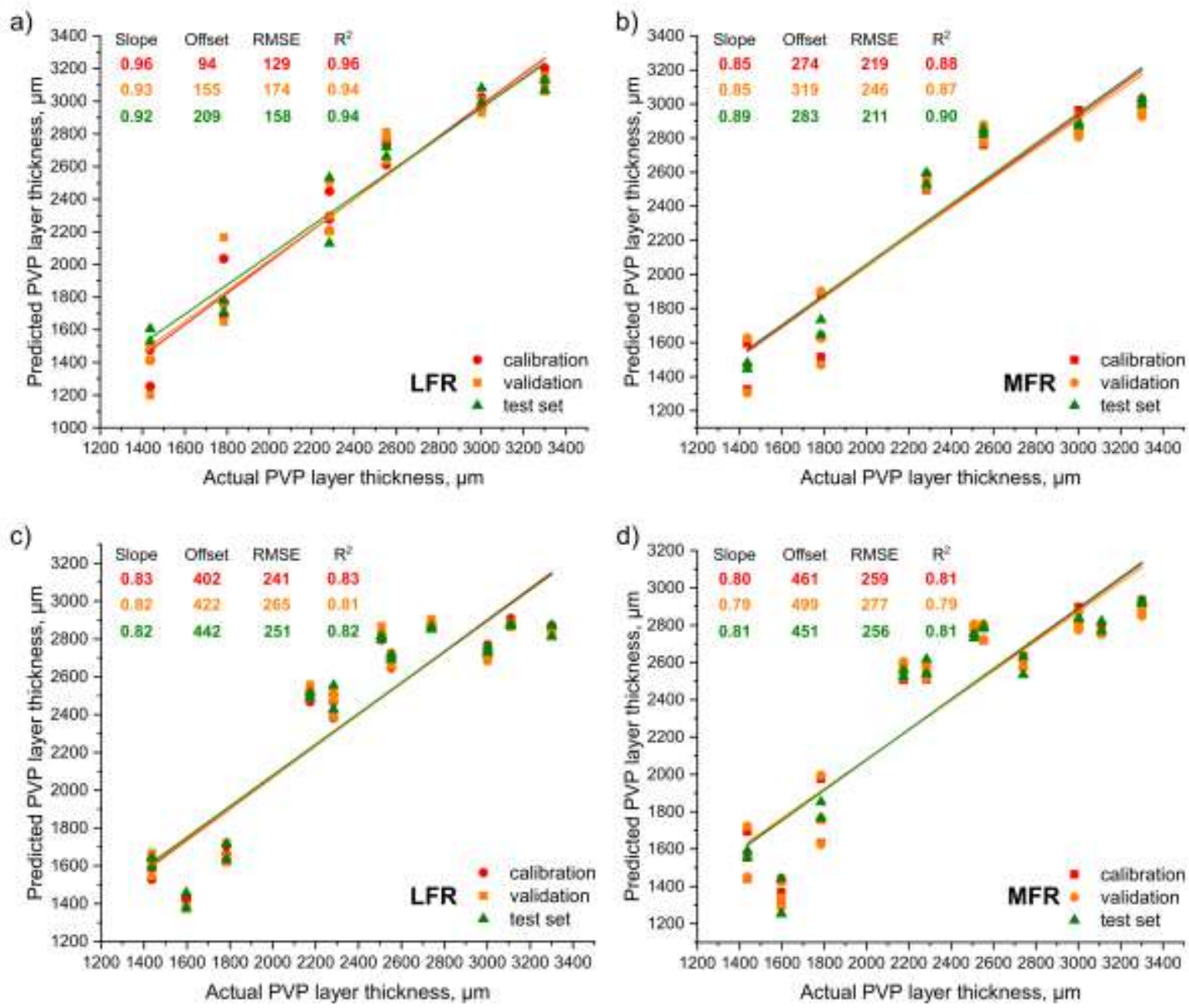

Figure S8. Calibration, cross validation and test set data of the selected PLS regression models using LFR and MFR data (left and right panels, respectively) for PVP layer thickness determination of bilayer tablets containing $(\mathrm{a}, \mathrm{b})$ constant celecoxib form III content $(100 \mathrm{mg})$ and $(\mathrm{c}, \mathrm{d})$ both constant and varied celecoxib form III content. 
Table S3. Summary of the performance for the PLS regression models created using either LFR or MFR micro-spatially offset spectroscopic data for bilayer tablets with constant celecoxib form III, but varied PVP content.

\begin{tabular}{|c|c|c|c|c|c|c|c|c|c|c|c|c|c|c|}
\hline & & \multicolumn{5}{|c|}{ Calibration } & \multicolumn{4}{|c|}{ Cross validation } & \multicolumn{4}{|c|}{ Test set } \\
\hline & Sample displacement, $\mathrm{mm}$ & Factors & Slope & Offset & RMSEC & $\mathrm{R}^{2}$ & Slope & Offset & RMSECV & $\mathrm{R}^{2}$ & Slope & Offset & RMSEP & $\mathrm{R}^{2}$ \\
\hline \multirow{11}{*}{ LFR } & 0 & \multirow{11}{*}{2} & 0.87 & 317 & 236 & 0.87 & 0.82 & 436 & 324 & 0.78 & 0.82 & 475 & 232 & 0.87 \\
\hline & 0.5 & & 0.86 & 330 & 241 & 0.86 & 0.82 & 428 & 325 & 0.77 & 0.81 & 480 & 249 & 0.85 \\
\hline & 1.0 & & 0.88 & 284 & 227 & 0.88 & 0.84 & 383 & 306 & 0.80 & 0.84 & 399 & 239 & 0.86 \\
\hline & 1.5 & & 0.88 & 278 & 221 & 0.88 & 0.85 & 362 & 301 & 0.81 & 0.85 & 403 & 220 & 0.88 \\
\hline & 2.0 & & 0.90 & 231 & 201 & 0.90 & 0.88 & 300 & 274 & 0.84 & 0.87 & 374 & 207 & 0.90 \\
\hline & 2.5 & & 0.90 & 230 & 201 & 0.90 & 0.87 & 300 & 270 & 0.84 & 0.92 & 219 & 199 & 0.91 \\
\hline & 3.0 & & 0.94 & 153 & 164 & 0.94 & 0.91 & 202 & 213 & 0.90 & 0.91 & 216 & 173 & 0.93 \\
\hline & 3.5 & & 0.94 & 144 & 159 & 0.94 & 0.92 & 194 & 206 & 0.91 & 0.89 & 256 & 181 & 0.92 \\
\hline & 4.0 & & 0.94 & 134 & 153 & 0.94 & 0.92 & 201 & 203 & 0.91 & 0.94 & 156 & 172 & 0.93 \\
\hline & 4.5 & & 0.96 & 94 & 129 & 0.96 & 0.93 & 155 & 174 & 0.94 & 0.92 & 209 & 158 & 0.94 \\
\hline & 5.0 & & 0.95 & 129 & 150 & 0.95 & 0.92 & 199 & 197 & 0.92 & 0.89 & 265 & 166 & 0.93 \\
\hline \multirow{11}{*}{ MFR } & $\mathbf{0}$ & \multirow{11}{*}{1} & 0.85 & 274 & 219 & 0.88 & 0.85 & 319 & 246 & 0.87 & 0.89 & 283 & 211 & 0.90 \\
\hline & 0.5 & & 0.87 & 313 & 234 & 0.87 & 0.85 & 357 & 261 & 0.86 & 0.83 & 416 & 240 & 0.86 \\
\hline & 1.0 & & 0.85 & 354 & 249 & 0.85 & 0.83 & 403 & 276 & 0.84 & 0.84 & 410 & 247 & 0.85 \\
\hline & 1.5 & & 0.85 & 348 & 247 & 0.85 & 0.83 & 397 & 274 & 0.84 & 0.87 & 336 & 252 & 0.85 \\
\hline & 2.0 & & 0.85 & 353 & 249 & 0.85 & 0.83 & 403 & 275 & 0.84 & 0.84 & 393 & 252 & 0.85 \\
\hline & 2.5 & & 0.84 & 388 & 261 & 0.84 & 0.81 & 443 & 289 & 0.83 & 0.85 & 383 & 247 & 0.85 \\
\hline & 3.0 & & 0.85 & 366 & 253 & 0.85 & 0.82 & 418 & 281 & 0.83 & 0.82 & 448 & 273 & 0.82 \\
\hline & 3.5 & & 0.85 & 367 & 253 & 0.85 & 0.82 & 420 & 281 & 0.83 & 0.83 & 400 & 268 & 0.83 \\
\hline & 4.0 & & 0.84 & 383 & 259 & 0.84 & 0.82 & 439 & 287 & 0.83 & 0.81 & 460 & 275 & 0.82 \\
\hline & 4.5 & & 0.84 & 384 & 259 & 0.84 & 0.81 & 440 & 289 & 0.82 & 0.83 & 410 & 281 & 0.81 \\
\hline & 5.0 & & 0.85 & 364 & 253 & 0.85 & 0.82 & 419 & 282 & 0.83 & 0.88 & 289 & 256 & 0.84 \\
\hline
\end{tabular}


Table S4. Summary of the performance for the global PLS regression models created using either LFR or MFR micro-spatially offset spectroscopic data for bilayer tablets with both constant and varied celecoxib form III content.

\begin{tabular}{|c|c|c|c|c|c|c|c|c|c|c|c|c|c|c|}
\hline & & \multicolumn{5}{|c|}{ Calibration } & \multicolumn{4}{|c|}{ Cross validation } & \multicolumn{4}{|c|}{ Test set } \\
\hline & Sample displacement, $\mathrm{mm}$ & Factors & Slope & Offset & RMSEC & $\mathrm{R}^{2}$ & Slope & Offset & RMSECV & $\mathrm{R}^{2}$ & Slope & Offset & RMSEP & $\mathrm{R}^{2}$ \\
\hline \multirow{11}{*}{ LFR } & 0 & \multirow{11}{*}{2} & 0.76 & 575 & 289 & 0.76 & 0.75 & 596 & 313 & 0.74 & 0.73 & 645 & 295 & 0.75 \\
\hline & 0.5 & & 0.76 & 590 & 292 & 0.76 & 0.75 & 610 & 316 & 0.73 & 0.74 & 634 & 295 & 0.75 \\
\hline & 1.0 & & 0.77 & 554 & 284 & 0.77 & 0.76 & 571 & 306 & 0.75 & 0.75 & 600 & 291 & 0.76 \\
\hline & 1.5 & & 0.78 & 537 & 279 & 0.78 & 0.77 & 554 & 302 & 0.75 & 0.76 & 591 & 288 & 0.76 \\
\hline & 2.0 & & 0.79 & 515 & 273 & 0.79 & 0.78 & 530 & 296 & 0.76 & 0.78 & 530 & 274 & 0.78 \\
\hline & 2.5 & & 0.80 & 489 & 266 & 0.80 & 0.79 & 507 & 289 & 0.78 & 0.79 & 515 & 273 & 0.79 \\
\hline & 3.0 & & 0.80 & 467 & 260 & 0.81 & 0.80 & 481 & 282 & 0.79 & 0.79 & 499 & 266 & 0.80 \\
\hline & 3.5 & & 0.81 & 456 & 257 & 0.81 & 0.80 & 472 & 280 & 0.79 & 0.80 & 485 & 263 & 0.80 \\
\hline & 4.0 & & 0.82 & 437 & 252 & 0.81 & 0.81 & 456 & 274 & 0.80 & 0.82 & 449 & 259 & 0.81 \\
\hline & 4.5 & & 0.84 & 394 & 239 & 0.84 & 0.83 & 413 & 262 & 0.82 & 0.82 & 436 & 253 & 0.82 \\
\hline & 5.0 & & 0.83 & 402 & 241 & 0.83 & 0.82 & 422 & 265 & 0.81 & 0.82 & 442 & 251 & 0.82 \\
\hline \multirow{11}{*}{ MFR } & $\mathbf{0}$ & \multirow{11}{*}{1} & 0.80 & 461 & 259 & 0.81 & 0.79 & 499 & 277 & 0.79 & 0.81 & 451 & 256 & 0.81 \\
\hline & 0.5 & & 0.80 & 471 & 261 & 0.80 & 0.79 & 506 & 278 & 0.79 & 0.78 & 538 & 272 & 0.79 \\
\hline & 1.0 & & 0.80 & 484 & 265 & 0.80 & 0.78 & 520 & 281 & 0.79 & 0.79 & 507 & 263 & 0.80 \\
\hline & 1.5 & & 0.81 & 462 & 259 & 0.81 & 0.79 & 497 & 275 & 0.80 & 0.81 & 461 & 265 & 0.80 \\
\hline & 2.0 & & 0.80 & 469 & 261 & 0.80 & 0.79 & 506 & 277 & 0.79 & 0.80 & 484 & 268 & 0.79 \\
\hline & 2.5 & & 0.79 & 494 & 268 & 0.79 & 0.78 & 533 & 284 & 0.78 & 0.80 & 486 & 261 & 0.80 \\
\hline & 3.0 & & 0.80 & 490 & 267 & 0.80 & 0.78 & 531 & 283 & 0.78 & 0.78 & 535 & 277 & 0.78 \\
\hline & 3.5 & & 0.81 & 465 & 260 & 0.81 & 0.79 & 505 & 276 & 0.79 & 0.79 & 486 & 271 & 0.79 \\
\hline & 4.0 & & 0.80 & 485 & 265 & 0.80 & 0.78 & 527 & 282 & 0.79 & 0.78 & 526 & 272 & 0.79 \\
\hline & 4.5 & & 0.81 & 467 & 260 & 0.81 & 0.79 & 510 & 277 & 0.79 & 0.80 & 486 & 275 & 0.78 \\
\hline & 5.0 & & 0.80 & 480 & 264 & 0.80 & 0.78 & 525 & 281 & 0.79 & 0.82 & 441 & 267 & 0.79 \\
\hline
\end{tabular}



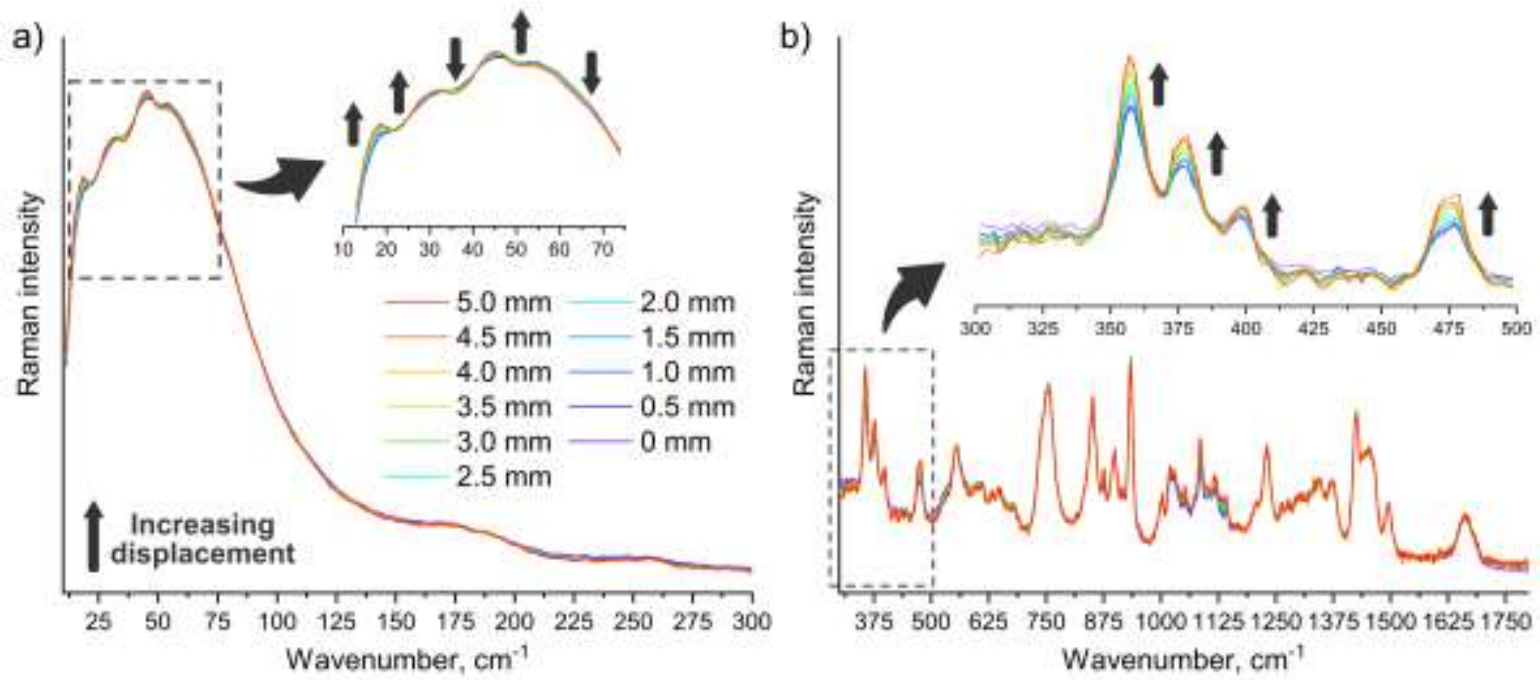

Figure S9. Examples of (a) low and (b) mid-frequency micro-spatially offset Raman spectra (SNV corrected for a better visibility of trends) for celecoxib form III, $\alpha$-LM and PVP trilayer tablet (1:2:1 composition; 50/100/50 mg). 
a)

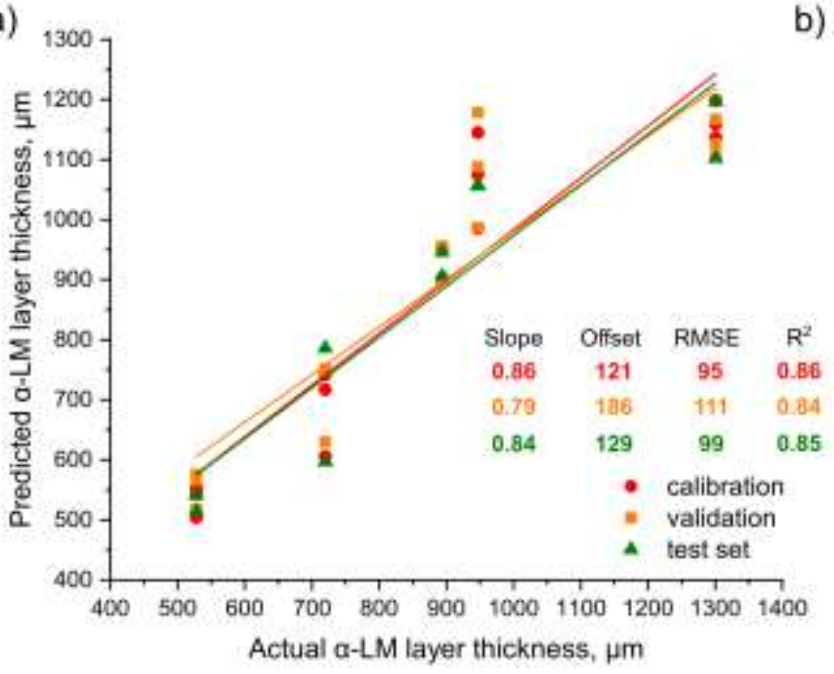

c)

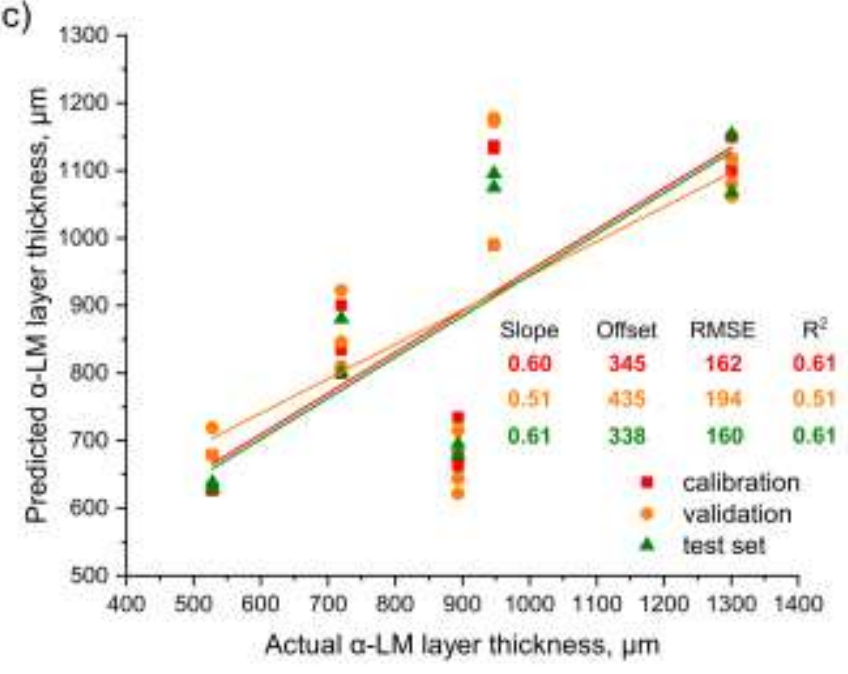

b)

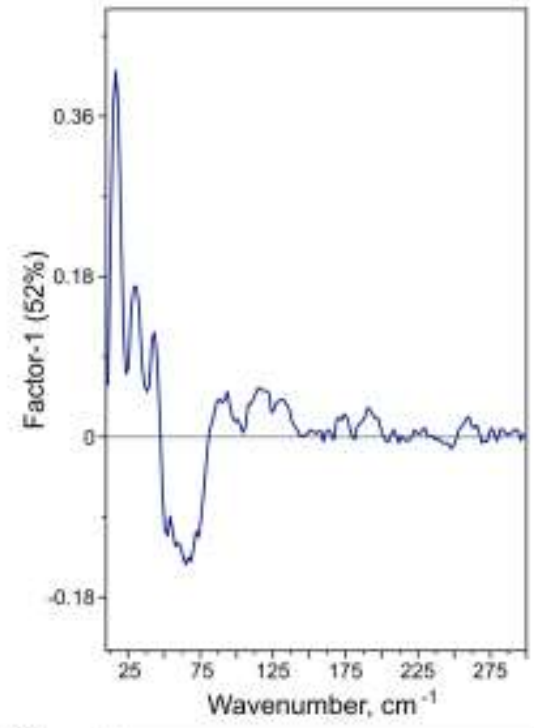

d)

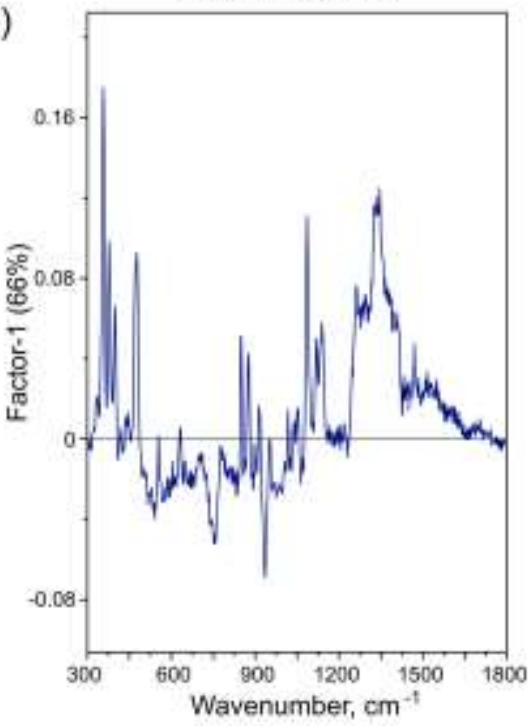

Figure S10. (a,c) Calibration, cross validation and test set data, and (b,d) loadings plots of respective factors of the selected PLS regression models using LFR and MFR data (upper and lower panels, respectively) for the middle ( $\alpha$-LM) layer thickness determination of $200 \mathrm{mg}$ trilayer tablets containing celecoxib form III, $\alpha$-LM and PVP. 
Table S5. Summary of the performance for the PLS regression models created using either LFR or MFR micro-spatially offset spectroscopic data for trilayer tablets with varied celecoxib form III, $\alpha$-LM and PVP content.

\begin{tabular}{|c|c|c|c|c|c|c|c|c|c|c|c|c|c|c|}
\hline & & \multicolumn{5}{|c|}{ Calibration } & \multicolumn{4}{|c|}{ Cross validation } & \multicolumn{4}{|c|}{ Test set } \\
\hline & Sample displacement, $\mathrm{mm}$ & Factors & Slope & Offset & RMSEC & $\mathrm{R}^{2}$ & Slope & Offset & RMSECV & $\mathrm{R}^{2}$ & Slope & Offset & RMSEP & $\mathrm{R}^{2}$ \\
\hline \multirow{11}{*}{ LFR } & 0 & \multirow{11}{*}{1} & 0.82 & 154 & 108 & 0.82 & 0.77 & 200 & 127 & 0.79 & 0.79 & 185 & 104 & 0.83 \\
\hline & 0.5 & & 0.83 & 153 & 108 & 0.83 & 0.77 & 200 & 126 & 0.79 & 0.80 & 161 & 101 & 0.85 \\
\hline & 1.0 & & 0.82 & 158 & 109 & 0.82 & 0.77 & 205 & 128 & 0.78 & 0.80 & 173 & 111 & 0.82 \\
\hline & 1.5 & & 0.82 & 161 & 110 & 0.82 & 0.77 & 208 & 130 & 0.78 & 0.76 & 208 & 116 & 0.80 \\
\hline & 2.0 & & 0.83 & 149 & 106 & 0.83 & 0.78 & 203 & 123 & 0.80 & 0.80 & 171 & 109 & 0.82 \\
\hline & 2.5 & & 0.83 & 153 & 107 & 0.83 & 0.77 & 209 & 126 & 0.79 & 0.82 & 153 & 103 & 0.84 \\
\hline & 3.0 & & 0.84 & 141 & 103 & 0.84 & 0.78 & 200 & 120 & 0.81 & 0.79 & 190 & 103 & 0.84 \\
\hline & 3.5 & & 0.86 & 126 & 98 & 0.86 & 0.79 & 186 & 113 & 0.83 & 0.85 & 136 & 102 & 0.84 \\
\hline & 4.0 & & 0.86 & 121 & 95 & 0.86 & 0.79 & 186 & 111 & 0.84 & 0.84 & 129 & 99 & 0.85 \\
\hline & 4.5 & & 0.85 & 127 & 98 & 0.85 & 0.78 & 204 & 113 & 0.83 & 0.84 & 158 & 104 & 0.84 \\
\hline & 5.0 & & 0.86 & 127 & 98 & 0.86 & 0.78 & 202 & 113 & 0.83 & 0.87 & 109 & 107 & 0.83 \\
\hline \multirow{11}{*}{ MFR } & $\mathbf{0}$ & \multirow{11}{*}{1} & 0.60 & 345 & 162 & 0.61 & 0.51 & 435 & 194 & 0.51 & 0.61 & 338 & 160 & 0.61 \\
\hline & 0.5 & & 0.53 & 414 & 177 & 0.53 & 0.42 & 508 & 211 & 0.41 & 0.54 & 409 & 173 & 0.55 \\
\hline & 1.0 & & 0.48 & 456 & 186 & 0.48 & 0.37 & 551 & 221 & 0.36 & 0.49 & 444 & 184 & 0.49 \\
\hline & 1.5 & & 0.45 & 479 & 190 & 0.45 & 0.35 & 570 & 226 & 0.33 & 0.43 & 496 & 212 & 0.39 \\
\hline & 2.0 & & 0.44 & 491 & 193 & 0.44 & 0.33 & 584 & 230 & 0.31 & 0.40 & 523 & 199 & 0.40 \\
\hline & 2.5 & & 0.41 & 512 & 197 & 0.41 & 0.30 & 609 & 236 & 0.27 & 0.40 & 526 & 200 & 0.40 \\
\hline & 3.0 & & 0.41 & 421 & 198 & 0.41 & 0.30 & 612 & 237 & 0.26 & 0.39 & 536 & 200 & 0.40 \\
\hline & 3.5 & & 0.40 & 532 & 199 & 0.40 & 0.30 & 616 & 237 & 0.26 & 0.41 & 513 & 200 & 0.39 \\
\hline & 4.0 & & 0.39 & 540 & 202 & 0.39 & 0.28 & 627 & 240 & 0.24 & 0.38 & 537 & 202 & 0.39 \\
\hline & 4.5 & & 0.37 & 556 & 205 & 0.37 & 0.27 & 641 & 243 & 0.22 & 0.36 & 558 & 206 & 0.36 \\
\hline & 5.0 & & 0.35 & 573 & 208 & 0.35 & 0.25 & 655 & 246 & 0.21 & 0.35 & 569 & 209 & 0.34 \\
\hline
\end{tabular}




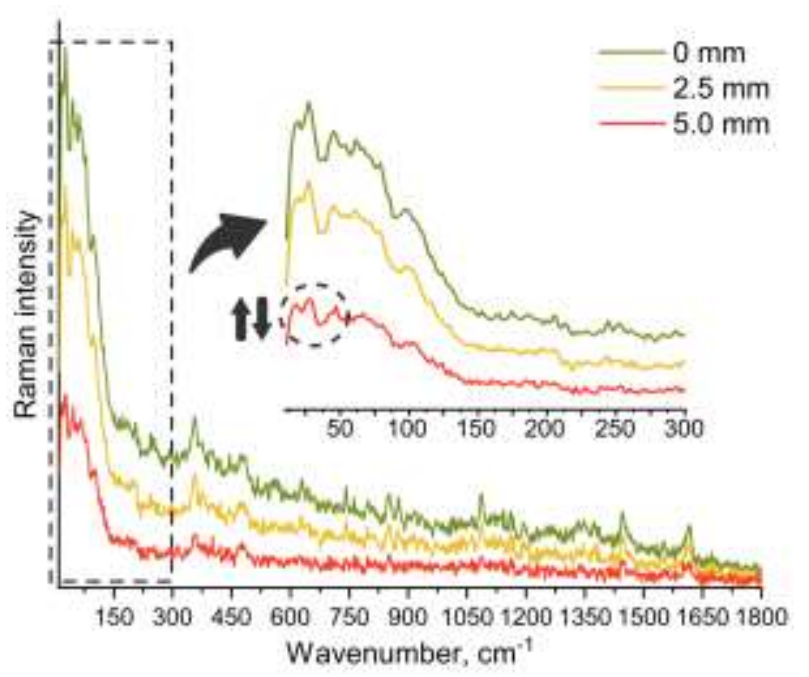

Figure S11. Examples of raw spectra collected at different sample displacements for the same areas of a multi-component homogenous tablet containing celecoxib, $\alpha$-LM and PVP dehydrated ( $\alpha$-LM to $\alpha-$ $\left.\mathrm{L}_{\mathrm{S}}\right)$ at $140{ }^{\circ} \mathrm{C}$ for 20 minutes.

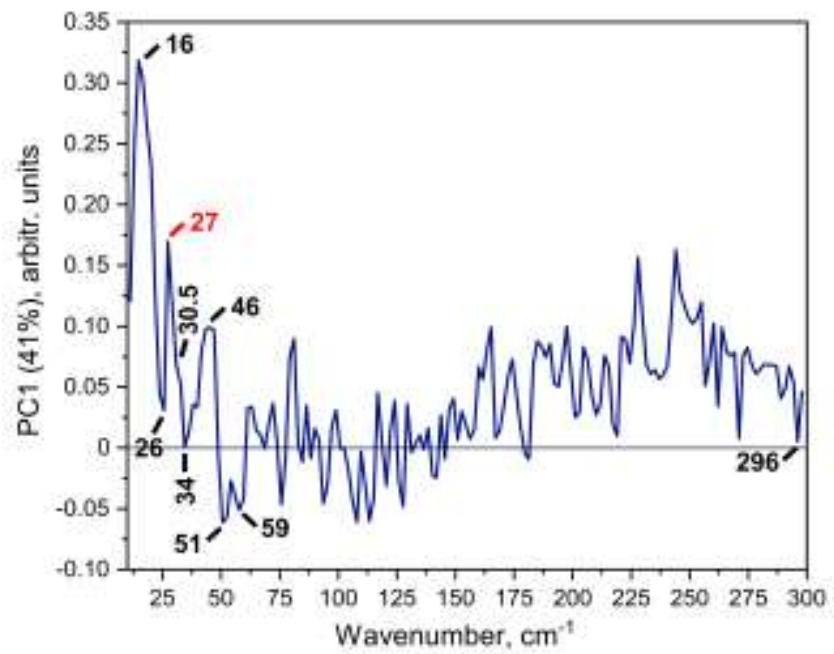

Figure S12. PC1 loadings plot for PCA of micro-SOLFRS mapping data collected from multicomponent tablet containing celecoxib, $\alpha$-LM and PVP dehydrated $\left(\alpha-\mathrm{LM}\right.$ to $\alpha$-Ls) at $140{ }^{\circ} \mathrm{C}$ for 20 minutes. The red labels denote unique peaks attributed to celecoxib form III. 